\title{
Self-Healing Hybrid Protection Architecture for Passive Optical Networks
}

\author{
Waqas A. Imtiaz \\ Department of Electrical Engineering \\ IQRA National University \\ Peshawar, Pakistan \\ P. Mehar \\ Department of Electrical Engineering \\ IQRA National University \\ Peshawar, Pakistan
}

\author{
M. Waqas \\ Department of Electrical Engineering \\ IQRA National University \\ Peshawar, Pakistan \\ Yousaf Khan \\ Department of Electrical Engineering \\ IQRA National University \\ Peshawar, Pakistan
}

\begin{abstract}
Expanding size of passive optical networks (PONs) along with high availability expectation makes the reliability performance a crucial need. Most protection architectures utilize redundant network components to enhance network survivability, which is not economical. This paper proposes new self-healing protection architecture for passive optical networks (PONs), with a single ring topology and star-ring topology at feeder and distribution level respectively. The proposed architecture provides desirable protection to the network by avoiding fiber duplication at both feeder and distribution level. Moreover, medium access control (MAC) controlled switching is utilized to provide efficient detection, and restoration of faults or cuts throughout the network. Analytical analysis reveals that the proposed self-healing hybrid protection architecture ensures survivability of the affected traffic along with desirable connection availability of $99.9994 \%$ at minimum deployment cost, through simple architecture and simultaneous protection against failures.
\end{abstract}

Keywords-passive optical network; protection; star-ring topology; reliability; CAPEX

\section{INTRODUCTION}

Passive optical network (PON) consists of a long feeder fiber (FF) between an optical line terminal (OLT) at the central office $(\mathrm{CO})$ and remote node $(\mathrm{RN})$ at the subscriber premises. RN contains an 1: $N$ optical coupler (CPR), which connects $N$ optical network units (ONUs) through dedicated distribution fibers (DFs). PONs are anticipated to solve the last mile bottleneck between high-speed core/metropolitan networks and access domain, owing to its significant advantages, like high subscribers count, minimum capital expenditure (CAPEX) due to shared FF, less operational expenditure (OPEX) due to passive components between OLT and ONU, and support for high data rates [1].

Efficient operation of PONs requires high availability along with fault detection and restoration for smooth transmission of data between OLT and subscriber premises [1-3]. Therefore, it is imperative for the network operators to develop simple and efficient protection architecture, which is reliable and economical for the common end user. Evolution of PON protection architectures began with ITU-T G.983.1 standards in the form of type A, B, C and D schemes. Type A, and B concentrates on protecting the feeder level only, while type C, and $\mathrm{D}$ protects the entire PON by duplicating components throughout the network. Consequently, type $\mathrm{A}$, and $\mathrm{B}$ fail to provide the desirable availability since no protection at provided at the distribution level. Whereas, type C and D provides high reliability performance, but unfortunately they require duplication of entire $\mathrm{PON}$, which significantly increase the overall capital expenditure (CAPEX) for the network that is shared among limited number of subscribers [2]. Moreover, it is also observed that use additional, redundant, components to provide the necessary protection reduce the network reliability [4].

Ring-based protection schemes including single and double ring architectures have been proposed to negate the issue of fiber duplication in PONs. In [5], a single ring based fiber is placed instead of the long FF, which changes into tree topology with two active branches in case of failure. However, the unprotected star shaped distribution network reduce its availability, and hence, feasibility for the access domain [3]. A similar single ring-star architecture is proposed in [6], with the use of relatively simple components. However, it also fails to provide the necessary protection to ONUs, which significantly reduces its reliability for access domain.

Ring based fault detection and restoration architecture for PONs is proposed in [7-8], which utilize CPRs for high availability of the network along with ring extension between multiple RNs. However, single CPR per ONU introduce serious power budget issues, which reduces the ability of single ring based PONs to support large number of subscribers [3]. Tree and star-ring architectures are proposed in [9-10] for protection at both feeder and distribution levels. However, duplication of FF between OLT and RN significantly increases the CAPEX, which is not viable for the common end user in access domain.

This paper proposes new hybrid protection architecture for PONs with a ring-star-ring topology, to ensure ubiquitous transmission at both feeder and distribution fibers. Moreover, 
MAC controlled switching add an efficient self-healing capacity to the proposed architecture. Operation of the proposed self-healing hybrid protection architecture is thoroughly analyzed for failures or cuts at both feeder and distribution fiber followed by reliability analysis through availability modeling technique in [3]. It is observed that the proposed system efficiently restores the flow the traffic in case of failure at both feeder and distribution fibers, along with the provision of desirable availability, 99.999\%, through simple architecture and simultaneous protection against failures.

\section{PROTECTION ARCHITECTURE}

The proposed self-healing hybrid protection architecture for PONs is shown in Fig. 1. All services are originating from OLT at the CO, which consists of a transmitter and receiver module. Output of OLT is connected to an optical circulator $\left(O C_{o c}\right)$, which splits the traffic for access domain and OLT receiver module respectively. Traffic from $O C_{o c}$ is fed into an erbium doped fiber amplifier (EDFA), which amplifies the optical signal to meet the power budget requirements of the proposed architecture. Output of EDFA is split into two identical paths through 1:2 $C P R_{c o}$. Path "a" of $C P R_{c o}$ extends the FF in the clockwise (CW) direction, while path " $b$ " in the counter clockwise (CCW) direction. Path " $b$ " also contains a 1:2 optical switch $\left(O S_{C o}\right)$, which activates in case of failure. Port 1 of $O S_{c o}$ is connected to the ground, while port 2 extends the FF in the CCW direction. Under normal mode of operation, $O S_{c o}$ is at position 1 , and OLT handles the traffic through path "a". In case of failure, OLT medium access control (MAC) layer flips the switch towards port 2, which immediately restores the flow of traffic. MAC controlled switching eliminates the needs of extra arrangement at OLT. Moreover, efficient algorithms can significantly eliminate delays and unnecessary switching in case of failures.
Feeder ring (FR) is formed by connecting both paths through a 2: 2 passive $C P R_{x 1}$ at $R N_{x}$ as shown in Fig. 1, where port 1 and port 3 of $C P R_{21}$ in $R N_{2}$ connect both paths of the FF to form a single ring topology at the feeder level. While port 2 and port 4 of $C P R_{21}$ extends the FR into the access arena through another 2: $M C P R_{x 2} \cdot C P R_{22}$ is used to connect $M$ number of ONUs with the FR through dedicated DFs as shown in Fig. 1. If $X$ represents the number of RNs, then the proposed system can support up to $X \times M$ number of ONUs.

Each ONU contains a 1:2 CPR with three ports as shown in Fig. 1. Port 1 is used to connect each ONU with its dedicated $\mathrm{DF}$, while port 2 is fed into $2: 1$ OS $_{\text {onu }}$. Port 3 of the CPR is used to form a ring at the distribution level (DR), which ensures ubiquitous supply of traffic in case of failure at the DFs through an efficient star-ring topology. By default, $O S_{\text {onu }}$ is at position 1 and all traffic is delivered through DFs. In case of failure at the DF, MAC layer of effected ONU changes the switch position, which immediately converts the flow of affected traffic from DF to the backup DR. Output of the $O S_{\text {onu }}$ is fed into an $O C_{\text {onu }}$, which distributes the traffic in both ONU receiver and transmitter modules.

\section{PROTECTION ANALYSIS}

\section{A. Feeder ring failure}

It is assumed that the proposed PON is under normal mode of operation and all traffic is delivered through path " $a$ ". If failure occurs at point "f" as shown in Fig. 2. OLT will not receive any traffic from ONUs placed beyond the point of failure $(\mathrm{PoF})$ and vice versa. Therefore, OLT will initiate its recovery mechanism and send DISCOVERY GATE (DG) packets to check the status of FR in terms of ONUs registration. If OLT receive registration messages from one or all ONUs of each RN, it will continue its normal operation through path "a".

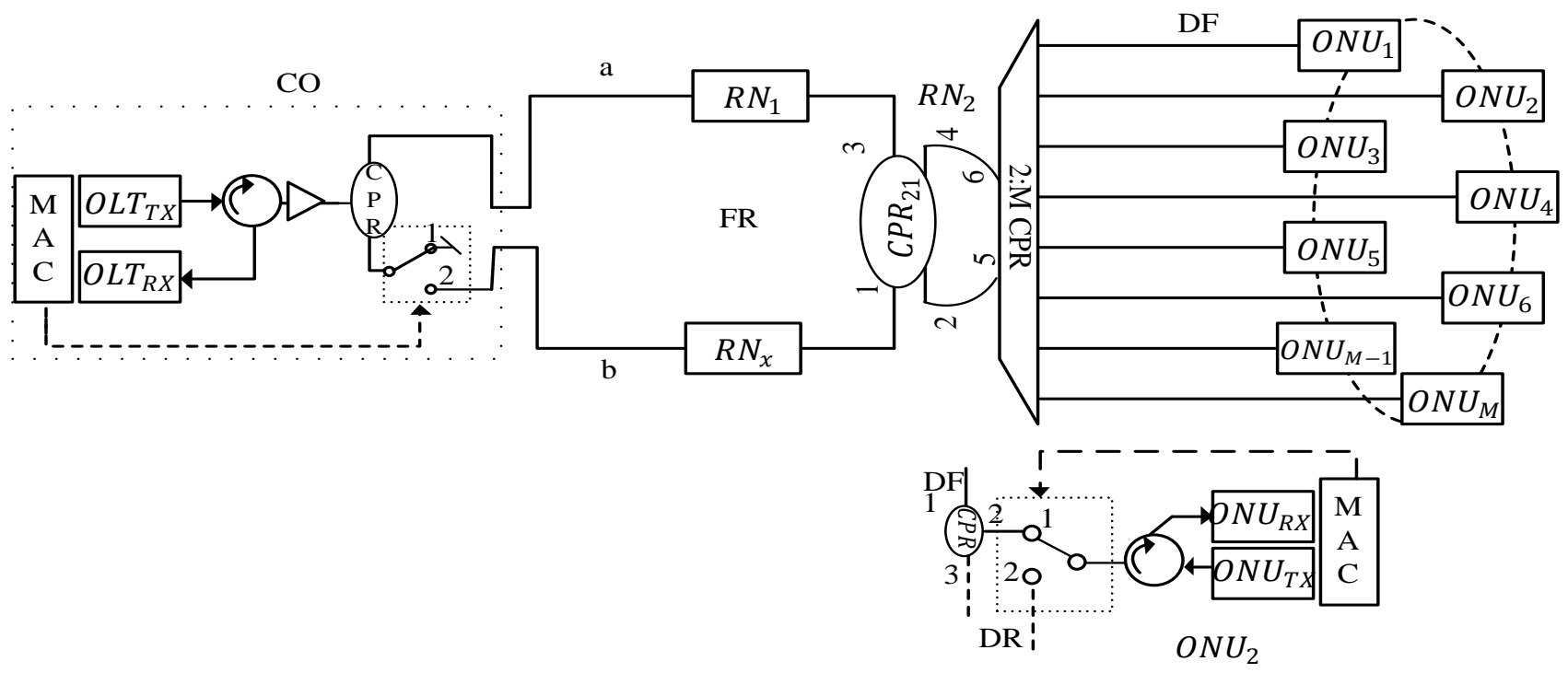

Fig. 1. Proposed self-healing hybrid PON

In case of negative registrations, OLT confirms any failure or cut across the FR through several atempts. On repeated negative registrations, OLT MAC layer will perform the switching operation, which converts the single ring topology into tree topology with two active branches as shown in Fig. 2. 
Path "a" will deliver traffic to ONUs before the PoF, while path " $b$ " will connect OLT with ONUs beyond the PoF.

It must be observed that ONUs will also initiate their recovery mechanism in case of failure at point " $\mathrm{f}$ ". In order to avoid any unnecessary switching the distribution level, the number of attempts for fault detection at the ONUs must be twice as compared to the number of attempts at OLT.

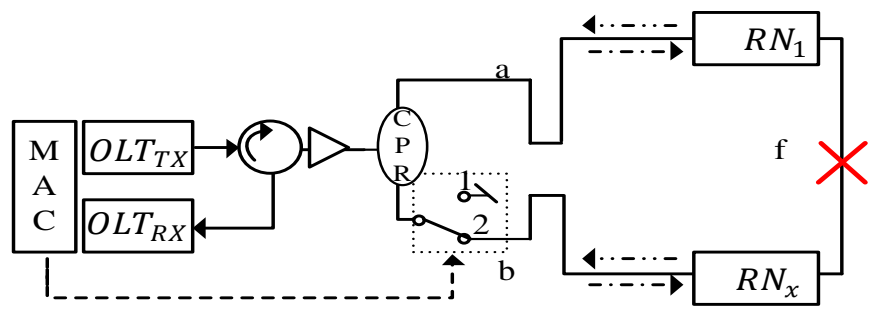

Fig. 2. Fault detection and recovery at FR

\section{B. Distribution fiber failure}

It is assumed that the $O S_{\text {onu }}$ is at position 1 , and $O N U_{2}$ sends and receive all traffic through the DF. If a failure or cut occurs at point "c", $O N U_{2}$ will stop to receive any traffic from the OLT as shown in Fig. 3. At the same time, OLT will cease to receive any upstream traffic from the disconnected $O \mathrm{NU}_{2}$. Thus, $\mathrm{ONU}_{2}$ will initiate its recovery mechanism, and send DG packets to check the status of DF. In case of successful registration messages from OLT, $\mathrm{ONU}_{2}$ will continue its normal operation through its respective DF. If no registration messages are received from OLT, ONU will attempt to confirm the fault or cut by sending several DG packets.

In case of successive negative registrations, $\mathrm{ONU}_{2} \mathrm{MAC}$ layer will flip the switch position to connect $O N U_{2}$ with the backup DR. Thus, all traffic will immediately transfer from the faulty $\mathrm{DF}$, to $\mathrm{ONU}_{3} \mathrm{DF}$ through $\mathrm{DR}$ between $O N U_{2}$ and $\mathrm{ONU}_{3}$.

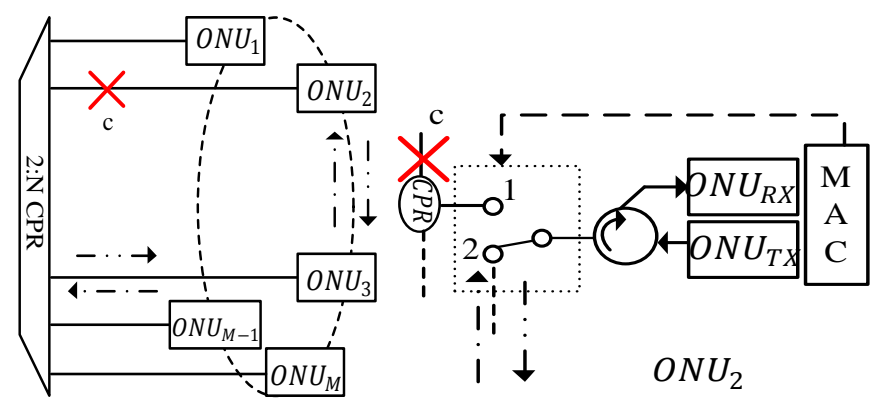

Fig. 3. Fault detection and restoration at DF

\section{ANALYTICAL ANALYSIS}

\section{A. Network capcity}

It is assumed that each $R N_{x}$ supports $M$ number of ONUs, then the total ONUs $N$ can be written as:

$$
N=X \times M
$$

If $\mathrm{L}$ represents the length of the fiber, $P_{C O}$ represents the power losses at the $\mathrm{CO}, P_{F R}$ represents the power losses at the FR including RNs, $P_{R X}$ is the power drop at the distribution network, and $R_{\text {sen }}$ represents the receiver sensitivity, then the downstream power budget can be written as:

$$
\begin{gathered}
P_{C O}=P_{T}-P_{O L T}-P_{O C}+P_{E D F A}-P_{C P R}-P_{O S} \\
P_{F R}=-\alpha L-X P_{C P R}-10 \log _{10}(M) \\
P_{R X}=-\alpha L-P_{C P R}-P_{O S}-P_{O C}-P_{O N U}
\end{gathered}
$$

Efficient operation of the proposed protection architecture requires that:

$$
P_{C O}+P_{F R}+P_{R X} \geq R_{\text {sen }}
$$

Now, rearranging (5) can determine the number of RNs, which can be written as:

$X \leq P_{T}-R_{\text {sen }}-16.25-10 \log _{10}(M)+P_{E D F A} / 3$

Table 1 shows the description and specifications of optical components in Eq. (2)-(4). Total number of ONUs, in the proposed PON are shown in Fig. 4, in relation to $M$ and $R_{\text {sen }}$ at $P_{T}=0 \mathrm{dBm}$, and $P_{E D F A}=25 \mathrm{~dB}$. It can be observed that the proposed protection architecture can support a large number of subscribers, owing to the use of 2:M CPR at RNs. This significantly reduce the power budget issue and allow the system to support maximum ONUs within power budget threshold.

TABLE I. OPTICAL COMPONENTS DESCRIPTION AND VALUES

\begin{tabular}{|c|l|c|}
\hline Symbols & Description & Values \\
\hline$P_{T}$ & Optical source power & $0 \mathrm{dBm}$ \\
\hline$P_{\text {OLT }}$ & Power loss at OLT & $3 \mathrm{~dB}$ \\
\hline$R_{\text {sen }}$ & Receiver sensitivity & $-20 \sim-24 \mathrm{dBm}$ \\
\hline$P_{\text {sa }}$ & Power loss at switch & $0.5 \mathrm{~dB}$ \\
\hline$P_{\text {edfa }}$ & Amplifier gain & $25 \mathrm{~dB}$ \\
\hline$P_{\text {oc }}$ & Circulator loss & $0.25 \mathrm{~dB}$ \\
\hline$P_{\text {onu }}$ & Power loss at ONU & $2 \mathrm{~dB}$ \\
\hline$\alpha$ & Propagation loss & $.25 \mathrm{~dB} / \mathrm{km}$ \\
\hline $10 \log _{10}(S)$ & Coupler loss (s=2) & $3 \mathrm{~dB}$ \\
\hline
\end{tabular}

\section{B. Reliability Analysis}

This section evaluates and compares reliability of the proposed architecture with protection schemes in [2], and [6] while using availability modeling technique in [3], and [11]. Figure 5 shows the reliability block diagrams (RBDs) of the protection architectures, where network components (including fibers) are arranged in series and parallel combination. Series arrangement refers to the unprotected components of the network. While, parallel combination represents the protected components of the network [3]. 


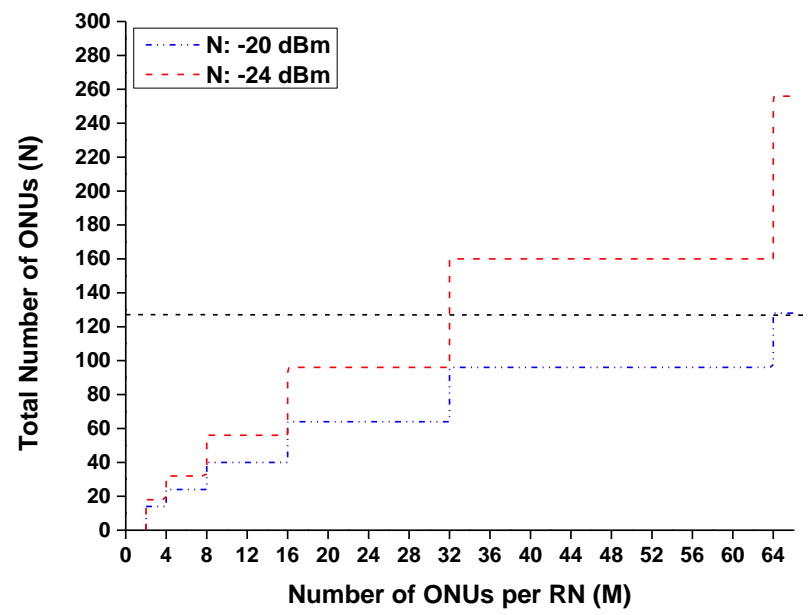

Fig. 4. Total number of ONUs at different values of $R_{\text {sen }}$

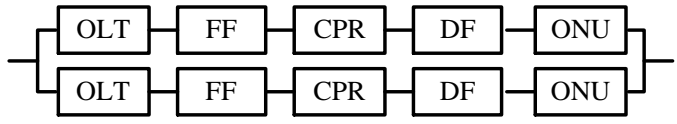

(a)

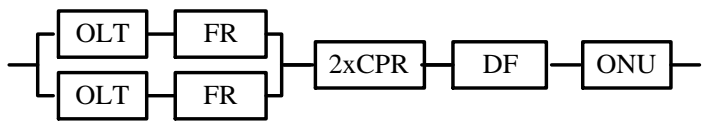

(b)

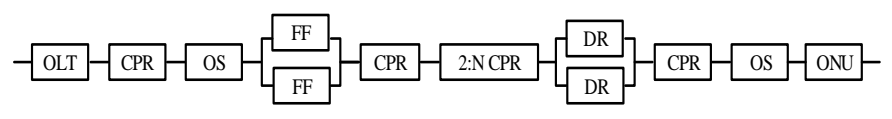

(c)

Fig. 5. Reliability block diagrams of different protection schemes: (a) scheme in [2] (b) scheme in [6] (c) proposed architecture

The characteristic parameter for each component in RBDs is the asymptotic unavailability $\left(U_{i}\right)$ where $i$ represent a component in the PON. Hence, the system availability with $m$ components is given by:

$$
A=1-\sum_{i=1}^{m} U_{i}
$$

Based on RBDs, availability of considered protection architecture can be written as:

$A_{[c]}=1-\left[\left(U_{O L T}+U_{F F}+U_{C P R}+U_{D F}+U_{O N U}\right) \times\right.$

$\left.\left(U_{O L T}+U_{F F}+U_{C P R}+U_{D F}+U_{O N U}\right)\right]$

$A_{[6]}=1-\left[\left(U_{O L T} \times U_{O L T}\right)+\left(U_{F R} \times U_{F R}\right)\right]+$

$\left.\left(2 \times U_{C P R}\right)+U_{D F}+U_{O N U}\right]$

$A_{P}=1-\left[U_{O L T}+U_{C P R}+U_{O S}+\left(U_{F F} \times U_{F F}\right)+\right.$

$U_{C P R}+U_{2: N C P R}+\left(U_{D F} \times U_{D F}\right)+U_{C P R}+U_{O S}+$ $\left.U_{\text {ONU }}\right]$

Connection aavailability is calculated for $20 \mathrm{~km}$ fiber at the feeder level and $5 \mathrm{~km}$ fiber at the distribution level, while using system unavailability parameters in Table 2 [3][11]. Figure 6 shows that type $\mathrm{C}$ protection scheme in [2] provides higher connection availability followed by our proposed architecture, $99.9994 \%$, which is above the minimum criteria for desirable connection availability (5 nines) [3]. While the similar ring-star topology in [6] fails to provide the desired reliability, due to its lack of protection at the distribution level.

Feasibility of the proposed architecture is further analyzed over CAPEX analysis, through components cost and analysis technique in [3]. Following parameters are adapted for fair comparison of the considered scheme. Tree based PONs contain $20 \mathrm{~km} \mathrm{FF}$ and $5 \mathrm{~km} \mathrm{DF}$, hybrid ring-star PONs contains $20 \mathrm{~km} \mathrm{FR,} 5 \mathrm{~km}$ DF and $1 \mathrm{~km}$ fiber between adjacent ONUs, single PON with 16 ONUs is considered in both tree and hybrid PONs, and two RNs are considered in hybrid ringbased PONs with eight ONUs each. Moreover, cost of fiber burying is ignored due to high variation. Cost comparison reveals that that our scheme provides desirable connection availability with minimum deployment cost of 2556 USD. Whereas, type $\mathrm{C}$ protection scheme duplicates the entire PON from OLT and ONU, which significantly increase their cost to 4218 USD per subscriber as compared to the proposed selfhealing hybrid protection architecture.

TABLE II. UNAVAILABILITY AND COST OF DifFERENT SySTEM ELEMENTS

\begin{tabular}{|l|c|}
\hline System Elements & Unavailability \\
\hline OLT (Tx and Rx) & $5.12 e^{-7}$ \\
\hline ONU (Tx and Rx) & $1.54 e^{-6}$ \\
\hline Optical Circulator & $3 e^{-7}$ \\
\hline Optical Switch & $1.2 e^{-6}$ \\
\hline RN (2:2 CPR) & $3 e^{-7}$ \\
\hline RN (2:N CPR) & $7.2 e^{-7}$ \\
\hline Fiber (/Km) & $1.37 e^{-5}$ \\
\hline
\end{tabular}

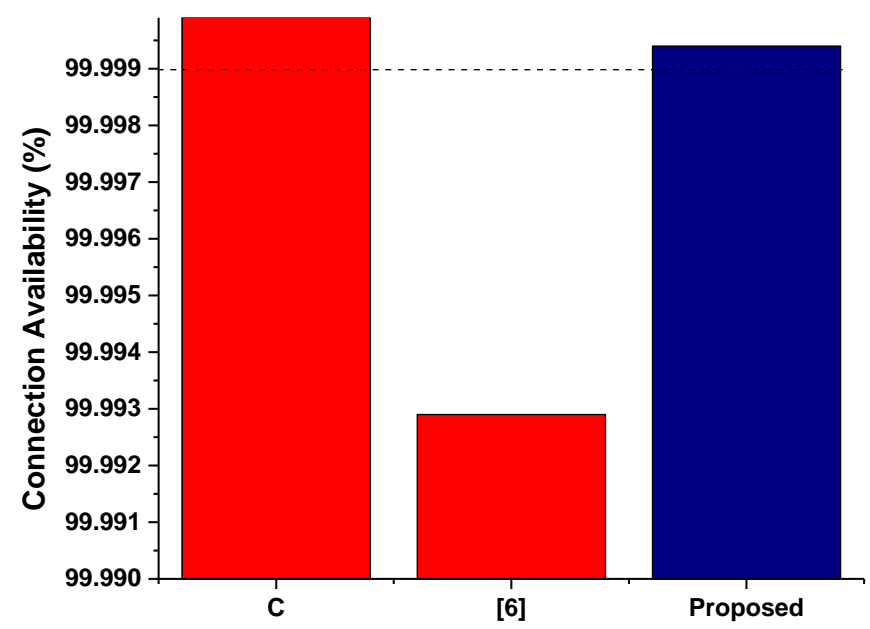

Protection Schemes

Fig. 6. Relaibility analysis of proposed scheme in comparison with different solutions

\section{CONCLUSION}

Efficient and economical protection architectures are characterized as an important factor for the adaption of PONs in access domain. This paper proposes new protection architecture for PONs, which ensures network survivability along with reduction in deployment cost. The proposed 
architecture consists of a single ring topology at the feeder level and a star-ring topology at the distribution level, which efficiently detects and restores the flow of affected traffic through MAC controlled switching. Moreover, it is observed through numerical analysis that the proposed self-healing hybrid protection architecture provides the desirable connection availability with no fiber duplication and hence less deployment cost as compare to the existing solutions.

\section{REFERENCES}

[1] C. Lam, Passive optical networks. Amsterdam: Elsevier/Academic Press, 2007.

[2] E. Wong, 'Survivable architectures for time and wavelength division multiplexed passive optical networks', Optics Communications, vol. 325, pp. 152-159, 2014.

[3] L. WOSINSKA, J. CHEN and C. LARSEN, 'Fiber Access Networks: Reliability Analysis and Swedish Broadband Market', IEICE Trans. Commun., vol. 92, no. 10, pp. 3006-3014, 2009.

[4] B. Lee, 'Simple ring-type passive optical network with two-fiber protection scheme and performance analysis', Optical Engineering, vol. 46, no. 6, p. 065002, 2007.

[5] Fu-Tai An, D. Gutierrez, Kyeong Soo Kim, Jung Woo Lee and L. Kazovsky, 'SUCCESS-HPON: A next-generation optical access architecture for smooth migration from TDM-PON to WDM-PON', IEEE Communications Magazine, vol. 43, no. 11, pp. S40-S47, 2005.

[6] X. Zhao, X. Chen, X. Fu, "A novel protection switching scheme for PONs with ring plus tree topology" Proc. SPIE 6022, Network Architectures, Management, and Applications III, 60223H December 05, 2005, doi:10.1117/12.636269.

[7] C. Yeh and S. Chi, 'Self-Healing Ring-Based Time-Sharing Passive Optical Networks', IEEE Photonics Technology Letters, vol. 19, no. 15, pp. 1139-1141, 2007.

[8] P. Lafata and J. Vodrážka, 'Experimental Verification of Passive Optical Network With Ring Topology', Microwave and Optical Technology Letters, vol. 55, no. 9, pp. 2201-2205, 2013.

[9] Y. Qiu and C. Chan, 'A novel survivable architecture for hybrid WDM/TDM passive optical networks', Optics Communications, vol. 312, pp. 52-56, 2014.

[10] C. Yeh, C. Chow and Y. Liu, 'Self-protected ring-star-architecture TDM passive optical network with triple-play management', Optics Communications, vol. 284, no. 13, pp. 3248-3250, 2011.

[11] M. Zhu, W. Zhong and S. Xiao, 'A Survivable Colorless Wavelength Division Multiplexed Passive Optical Network With Centrally Controlled Intelligent Protection Scheme', J. Opt. Commun. Netw., vol. 4 , no. 10 , p. $741,2012$. 